\title{
Doença Hipertensiva Específica da Gestação: conhecimentos de um grupo de gestantes usuárias de uma Unidade Básica de Saúde
}

\author{
Pregnancy-specific Hypertensive Disease: knowledge of a group of pregnant women users of a \\ Basic Health Unit \\ Enfermedad Hipertensiva Específica del Embarazo: conocimiento de un grupo de mujeres \\ embarazadas que utilizan una Unidad Básica de Salud
}

Recebido: 21/01/2022 | Revisado: 26/01/2022 | Aceito: 06/02/2022 | Publicado: 11/02/2022

\author{
Bianca Kaline Ferreira Torres \\ ORCID: https://orcid.org/0000-0003-2792-1398 \\ Universidade Maurício de Nassau, Brasil \\ E-mail: biancakaline10@gmail.com \\ Acsa Allynny dos Santos Silva \\ ORCID: https://orcid.org/0000-0002-2820-8650 \\ Universidade Maurício de Nassau, Brasil \\ E-mail: acsaallynny17@gmail.com \\ Joelma Maria Silva de Souza \\ ORCID: https://orcid.org/0000-0002-2972-3531 \\ Universidade Maurício de Nassau, Brasil \\ E-mail: jmtet@hotmail.com \\ Gabrielly Laís de Andrade Souza \\ ORCID: https://orcid.org/0000-0003-2078-9574 \\ Universidade Maurício de Nassau, Brasil \\ E-mail: gabriellylais18@gmail.com \\ Mariana Batista da Silva \\ ORCID: https://orcid.org/0000-0003-2015-9927 \\ Prefeitura Municipal de Gravatá, Brasil \\ E-mail: marianabatistas@outlook.com
}

\begin{abstract}
Resumo
A Doença Hipertensiva Específica da Gestação (DHEG) é uma doença multissistêmica que acomete de 5\% a $17 \%$ das gestações, o que causa diversas complicações resultando em significativa morbimortalidade materna e fetal. Nesse estudo, objetivou-se identificar os conhecimentos referentes a DHEG, portados pelas gestantes usuárias da Unidade Básica de Saúde (UBS) Cuiabá, localizada na cidade de Joaquim Nabuco, no estado de Pernambuco. Trata-se de um estudo do tipo descritivo, de corte transversal com abordagem qualitativa, que ocorreu por meio de entrevista, onde participaram 20 gestantes. Entre as gestantes entrevistadas, verificou-se idade média de aproximadamente 26 anos e baixo nível de escolaridade. Apresentaram conhecimento sucinto, com respostas curtas e sensação de dúvida ao responder, no tocante, apenas 04 gestantes afirmaram terem sido orientadas quanto à doença no pré-natal. Notou-se resultados superficiais frente as noções básicas desta patologia pelas gestantes, bem como a atuação do profissional frente à promoção de saúde.
\end{abstract}

Palavras-chave: Atenção primária à saúde; Educação em saúde; Pré-eclâmpsia; Conhecimento.

\begin{abstract}
Pregnancy-Specific Hypertensive Disease (PSHD) is a multisystem disease that affects $5 \%$ to $17 \%$ of pregnancies, which causes several complications resulting in significant maternal and fetal morbidity and mortality. In this study, the objective identifying the knowledges related to PSHD, carried by pregnant women users of the Basic Health Unit (BHU) Cuiabá, located in the city of Joaquim Nabuco, in the state of Pernambuco. It's about a descriptive search, cross-sectional study with a qualitative approach, occurred through interviews, in which 20 pregnant women participated. Among the pregnant women interviewed, there was an average age of approximately 26 years and a low level of education. They presented succinct knowledge, with short answers and a feeling of doubt when answering, with regard to only 04 pregnant women said they had been oriented about the disease in the prenatal period. Superficial results were noted in relation to the basic notions of this pathology by pregnant women, as well as the professional's performance in terms of health promotion.
\end{abstract}

Keywords: Primary health care; Health education; Pre-eclampsia; Knowledge. 


\begin{abstract}
Resumen
La Enfermedad Hipertensiva Específica del Embarazo (EHGH) es una enfermedad multisistémica que afecta del 5\% al $17 \%$ de los embarazos, que causa varias complicaciones que resultan en una importante morbilidad y mortalidad materna y fetal. En este estudio, el objetivo fue identificar los conocimientos relacionados con la EHGH, que llevan las embarazadas usuarias de la Unidad Básica de Salud (UBS) Cuiabá, ubicada en la ciudad de Joaquim Nabuco, en el estado de Pernambuco. Se trata de un estudio descriptivo, transversal, con abordaje cualitativo, que se realizó mediante entrevistas, en el que participaron 20 gestantes. Entre las mujeres embarazadas entrevistadas, hubo una edad promedio de aproximadamente 26 años y un bajo nivel de educación. Presentaron conocimientos sucintos, con respuestas breves y un sentimiento de duda al contestar, con respecto a solo 04 mujeres embarazadas que dijeron haber sido orientadas sobre la enfermedad en el período prenatal. Se observaron resultados superficiales en relación a las nociones básicas de esta patología por parte de la gestante, así como al desempeño del profesional en términos de promoción de la salud.
\end{abstract}

Palabras clave: Atención primaria de salud; Educación para la salud; Preeclampsia; Conocimiento.

\title{
1. Introdução
}

A hipertensão arterial sistêmica (HAS) é um preocupante agravo para a saúde pública brasileira e mundial. Caracteriza-se por níveis pressóricos elevados $\geq 140$ e/ou $90 \mathrm{mmHg}$, e é considerada uma condição clínica multifatorial que se associa, regularmente, a alterações funcionais e/ou estruturais dos órgãos-alvo e a distúrbios metabólicos. No Brasil, sua prevalência varia entre $22 \%$ e $44 \%$ em adultos (32\% em média), atingindo $65 \%$ para indivíduos acima de 60 anos e chegando a $71,7 \%$ em indivíduos acima de 70 anos (SBC, 2016; SBC, 2021).

Segundo Leal et al. (2018), em 2015, 20,7\% dos óbitos no Brasil aconteceram em razão dos transtornos hipertensivos durante o período gestacional, o parto e puerpério; $17,5 \%$ por problemas no trabalho de parto; e, $13,2 \%$ por complicações prevalentemente puerperais.

Quando presente na gestação, o que acomete de 5\% a 17\%, a hipertensão causa diversas complicações provocando significativa morbimortalidade materna e fetal. Dentre essas complicações, encontra-se a Doença Hipertensiva Específica da Gestação (DHEG), também chamada de Pré-Eclâmpsia. Esta, é definida como uma doença multissistêmica caracterizada pela presença da HAS relacionada com a proteinúria, após a $20^{\circ}$ semana de gestação e o seu desaparecimento até 12 semanas após o parto (Brasil, 2016; Antunes et al., 2017).

A etiologia da DHEG não é claramente conhecida. Abrahão et al. (2020), consideram que haja uma associação entre fatores genéticos, ambientais e imunológicos, que corrobora para uma invasão trofoblástica defeituosa das artérias espiraladas. Esse defeito acarreta na diminuição da perfusão uteroplacentária ocasionando em isquemia da placenta. Essa isquemia libera fatores, que dão início a uma cascata de eventos celulares e moleculares, causando disfunção do endotélio, o que leva a um aumento da resistência vascular e pressão arterial.

Sugere-se também que fatores maternos referentes à predisposição genética, à adequação imunológica à gestação e patologias vasculares pré-existentes estejam abrangidos nessa doença. Em razão disso, o tratamento definitivo para o controle dos sintomas da DHEG, é o início do parto e a remoção completa da placenta (Silva et al., 2021).

A DHEG é influenciada por fatores de risco não modificáveis, como idade, cor, hereditariedade, primiparidade; e por fatores de risco modificáveis, como sedentarismo, obesidade, tabagismo, HAS, Diabetes mellitus (DM), nível sócio econômico, extremos de idade reprodutiva, entre outros, que refletem nos hábitos e no estilo de vida (Oliveira et al., 2016)

Thuler et al. (2018) no contexto do sistema único de saúde (SUS), caracterizam a Atenção Primária à Saúde (APS) como o primeiro nível de atendimento e tem como propósito acolher as gestantes, privilegiando ações de promoção, proteção e recuperação da saúde, de forma integral e continuada. Nas Unidades básicas de Saúde (UBS), é realizado o pré-natal, que consiste no acompanhamento da gestante, durante todo o período gravídico, pela equipe multiprofissional de saúde.

Para Cassiano et al. (2020), durante o pré-natal, há, entre os profissionais de saúde e a gestante, uma relevante interação, proporcionando uma efetivação na promoção à saúde. Também são identificados fatores de riscos, onde devem 
atentar para a prevenção, diagnóstico precoce e educação em saúde, reduzindo assim as complicações da mãe e do feto. Assim, o pré-natal estabelece uma indispensável oportunidade para orientar e fornecer conhecimentos às gestantes sobre as diversas complicações da gravidez, entre elas a Pré-Eclâmpsia (Araújo et al., 2017).

Antônio, Pereira e Galdino (2019) apontam que os conhecimentos que as gestantes possuem a respeito das complicações que podem ser sujeitas, é insuficiente, e, para que possam compreender melhor o processo em que vivenciam, é necessário que tenham acesso as ações de educação em saúde, onde serão esclarecidas de forma eficaz.

Conforme Santana et al. (2019), de modo geral, as gestantes tem uma percepção de que a DHEG é apenas uma doença de riscos, havendo conhecimentos minuciosos somente mediante internação hospitalar pela gravidade clínica a que se encontram. Desta forma, torna-se importante a transmissão de conhecimentos para essas gestantes por parte dos profissionais de saúde durante o pré-natal nas UBS, despertando-as para o autocuidado.

Diante do exposto, e considerando a relevância do tema no contexto da saúde da mulher, a pesquisa surgiu com a necessidade de identificar os conhecimentos e percepções das gestantes sobre a doença. Tendo em vista estudos semelhantes, onde relatam que o pouco conhecimento que as gestantes possuem referente à DHEG nesse período é insuficiente para prevenir os agravos na saúde causados pela mesma. Dessa forma, este estudo teve como questão norteadora: quais os conhecimentos, que as gestantes usuárias da Unidade Básica de Saúde (UBS) Cuiabá, tem sobre a DHEG?

O estudo tem como objetivo identificar os conhecimentos referentes a DHEG, portados pelas gestantes usuárias da UBS Cuiabá, localizada na cidade de Joaquim Nabuco, no estado de Pernambuco, no intento de contribuir de forma incentivadora o meio acadêmico e possibilitar aos profissionais de saúde, elaboração de estratégias de educação em saúde que intencionem a disseminação de conhecimentos para as gestantes.

\section{Metodologia}

Trata-se de um estudo do tipo descritivo de corte transversal com abordagem qualitativa (Gil, 2017; Aragão, 2011; Silva et al.,2014), que buscou identificar os conhecimentos das gestantes sobre a DHEG, o qual foi desenvolvido na UBS Cuiabá, localizada na cidade de Joaquim Nabuco, território da Zona da Mata Sul, do estado de Pernambuco, em setembro e outubro de 2021 sob a supervisão dos pesquisadores e da enfermeira da unidade.

A pesquisa foi realizada em duas etapas, sendo a primeira destinada à realização de reuniões de acolhimento, que tiveram o objetivo de esclarecer a finalidade da pesquisa e os procedimentos que seriam adotados para coleta de dados, e também, a realização do convite para participar da pesquisa, enquanto a segunda, foi destinada a assinatura e concordância do Termo de Consentimento Livre e Esclarecido (TCLE) e aplicação do instrumento de coleta de dados.

Dessa forma, a coleta de dados foi realizada através de entrevista individualizada, em sala fechada, com base em um questionário aberto, composto por 16 questões. Teve duração máxima de 30 minutos, de acordo com a disponibilidade da gestante e utilizou como meio de coleta a gravação de áudio. A coleta foi realizada pela pesquisadora responsável e uma pesquisadora colaboradora, onde uma fez a entrevista e a outra fez anotações em diário de bordo.

Participaram da pesquisa, 20 gestantes, as quais responderam o instrumento de coleta de maneira livre e espontânea. E, os dados coletados, foram analisados e interpretados através da técnica de Laurence Bardin, que tem o objetivo de examinar diferentes subsídios de conteúdo, sejam eles verbais ou não-verbais.

Utilizou-se como critérios de inclusão, gestantes que tinha 18 anos ou mais; que estavam no primeiro ou segundo trimestre de gestação; cadastradas na UBS Cuiabá; e, utilizadoras do pré-natal da unidade. Excluíram-se, gestantes com grau de parentesco familiar com algum membro da equipe responsável pela pesquisa e que apresentaram problemas cognitivos que as inviabilizaram de responder o instrumento de coleta de forma adequada.

Por fim, vale ressaltar que a pesquisa foi aprovada pelo Comitê de Ética e Pesquisa Envolvendo Seres Humanos do 
Centro Universitário do Vale do Ipojuca - CEP | UNIFAVIP/UNIFBV | Wyden, sob o parecer n 4.971.067, e que em todas as etapas da pesquisa foi assegurado às gestantes o anonimato, a confidencialidade dos dados pessoais e a utilização das informações apenas para fins científicos, atendendo a Resolução 510/16 e 466/12 do Conselho Nacional de Saúde.

\section{Resultados e Discussão}

Compreender os conhecimentos das gestantes acerca da Doença Hipertensiva Específica da Gestação (DHEG) é de extrema relevância, visto que é constatado que a investigação sobre o entendimento que estas gestantes possuem em relação ao processo gestacional, ao estado de saúde e às situações de complexidade que podem estar sujeitas, é limitada (Silva et al., 2011).

Deste modo, com o intuito de buscar entender os conhecimentos das gestantes quanto à DHEG, foi realizada uma pesquisa em uma Unidade Básica de Saúde (UBS), localizada na cidade de Joaquim Nabuco, Zona da Mata Sul de Pernambuco. Na qual, participaram 20 gestantes. As mesmas, quando agrupadas pelas variáveis de idade, escolaridade, estado civil e portadoras de Hipertensão Arterial Sistêmica (HAS), apresentaram-se conforme ilustrado na Tabela 1.

Tabela 1: Distribuição das participantes de acordo com as variáveis de idade, escolaridade, estado civil e portadoras de HAS.

\begin{tabular}{c|c|c|c}
\multirow{2}{*}{ Variáveis } & Categoria & $\mathbf{N}^{\mathbf{0}}$ & \% \\
\hline \multirow{2}{*}{ Idade } & Entre 18 e 28 anos & 15 & $75 \%$ \\
& Entre 30 e 35 anos & 5 & $25 \%$ \\
\hline \multirow{2}{*}{ Escolaridade } & Ensino Fundamental Incompleto & 6 & $14 \%$ \\
& Ensino Fundamental Completo & 3 & $24 \%$ \\
& Ensino Médio Incompleto & 5 & $24 \%$ \\
& Ensino Médio Completo & 5 & $9 \%$ \\
\hline \multirow{2}{*}{ Estado Civil } & Ensino Superior Completo & 2 & $70 \%$ \\
& Solteira & 14 & $30 \%$ \\
\hline \multirow{2}{*}{ Portadora de HAS } & Casada & 6 & $10 \%$ \\
\hline
\end{tabular}

Fonte: Autores (2021).

De acordo com a Tabela 1, em relação à idade, identificou-se que a faixa etária média das participantes foi de aproximadamente 26 anos, o que condiz com a idade fértil reprodutiva das mulheres brasileiras, sendo de 10 a 49 anos (Brasil, 2011).

No que concerne à escolaridade, nota-se que, no geral, as participantes possuem um baixo nível, o que remete à maior dificuldade de acesso à informação e aos cuidados de saúde fundamentais para o período gravídico, favorecendo assim um déficit do autocuidado (Guimarães, 2014).

Em continuação na análise dos dados, as gestantes foram identificadas de acordo com a apresentação das falas pela letra "G" e numeradas conforme a ordem das entrevistas. 
Após transcrição dos áudios das entrevistas, foi realizada leitura detalhada, procurando os pontos semelhantes e divergentes dos dados coletados, e com isso foram idealizadas duas categorias de análise: Conhecimentos das gestantes acerca da DHEG e Transmissão de informações no pré-natal.

\subsection{Conhecimentos das gestantes acerca da DHEG}

Para algumas gestantes, a DHEG pode não retratar um problema que exige cuidado e controle, uma vez que a carência de conhecimento a respeito das complicações da doença é uma realidade para muitas delas (Jacob et al., 2021).

Durante a entrevista, quando questionadas sobre o conhecimento a respeito da Pré-eclâmpsia, apenas três (15\%) gestantes relataram nunca ter ouvido falar sobre a doença, conforme ilustrado no Gráfico 1:

Gráfico 1: Conhecimento acerca da Pré-eclâmpsia.

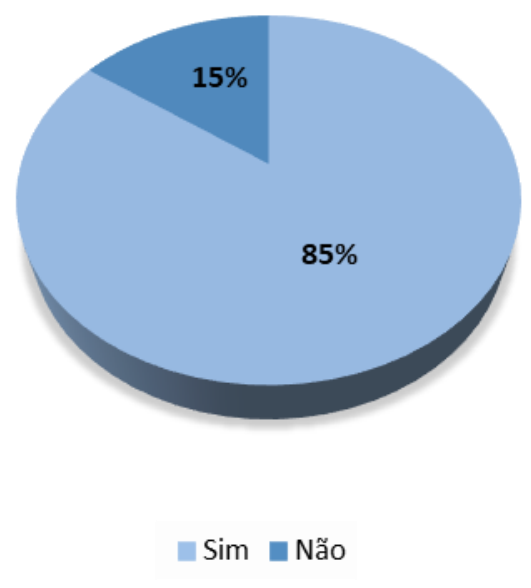

Fonte: Autores (2021).

Porém, quando indagadas sobre o conceito e complicações da doença, percebeu-se, pelas respostas obtidas e pela expressão facial, registrada na observação durante a entrevista, um entendimento sucinto, com respostas curtas e sensação de dúvidas ao responder, como mostra as falas descritas abaixo:

G1 - "Eu não sei/ eu não sei explicar, é uma doença que pode complicar na gestação e pode afetar o bebê"”

G12 - "A mãe pode ficar sem enxergar, sem andar, sem movimentar as pernas e os braços"

G10 - "É quando a mulher passa da hora de ter o bebê"

G14 - "Não sei explicar, é arriscado a vida da pessoa e da criança"

G6 - "Já ouvi falar, mas não entendo o que é"

Percebe-se então, que essas gestantes possuem um conhecimento reduzido e insegurança quanto aos saberes. Esse déficit de conhecimento mostrado pelas falas das entrevistadas, reflete na negligência pelo autocuidado, acarretando, algumas vezes, em hospitalização e/ou parto precoce. Pois, a menor consciência a respeito da DHEG, afetará na procura prévia pelos serviços de saúde por parte da gestante (Teixeira et al., 2017; Berhe et al., 2020).

Isso, reporta-se à necessidade de serem elaboradas medidas de aprimoramento na qualidade de saúde e expansão da assistência durante a gestação (Jacob et al., 2018).

Por outro lado, algumas gestantes entrevistadas mostraram possuir conhecimentos consideráveis acerca do conceito e complicações da doença, como expostos nas falas a seguir: 
G16 - "O bebê pode nascer com complicações, pode ir a óbito e a mãe também"

G20 - "Pressão arterial e proteína na urina, pode ter complicações na gravidez trazendo risco de morte para a mãe e o bebê"

Essas gestantes que expuseram esses conhecimentos sobre a DHEG, são mulheres que concluíram o ensino superior, o que vai de encontro a um estudo realizado na Etiópia, que mostra que em comparação com gestantes de baixo nível educacional, gestantes com ensino superior completo e com elevado nível de escolaridade tinha 4,9 vezes mais chances de saberem sobre essa patologia. Isso se dá devido à maior facilidade de acesso à informação, educação e comunicação (Berhe et al., 2020).

Segundo Antônio, Pereira e Galdino (2019), o conhecimento insuficiente das gestantes acarreta em um tratamento mais lento, ineficaz e prejudicado. Isso acontece por não haver uma busca de informações por parte da gestante, durante a assistência prestada pelos profissionais de saúde, e estes, não se preocupar com essa lacuna. A falta dessas informações pode implicar em indiligências diante de tal responsabilidade, no que concerne na prevenção e tratamento da doença (Almeida \& Souza, 2016).

Para tanto, é necessário que haja um comprometimento com o autocuidado, objetivando em melhoria da saúde e bemestar, e garantia de estilo de vida saudável para as mulheres. Isso é possível mediante uma assistência mais efetiva e eficiente, favorecendo a socialização de conhecimentos (Mota et al., 2021).

\subsection{Transmissão de informações no pré-natal}

A falta de informação das mulheres grávidas é um dos fatores potenciais para o atraso na busca de atendimento e decisão e um gargalo para o diagnóstico precoce e tratamento de doenças que podem surgir nesse período (Berhe et al., 2020).

Durante a entrevista, quando interrogadas se no decorrer do pré-natal foi falado sobre a Pré-eclâmpsia, apenas quatro gestantes relataram ter sido orientadas quanto a doença, como mostra o Gráfico 2:

Gráfico 2: Orientações sobre a Pré-eclâmpsia no pré-natal.

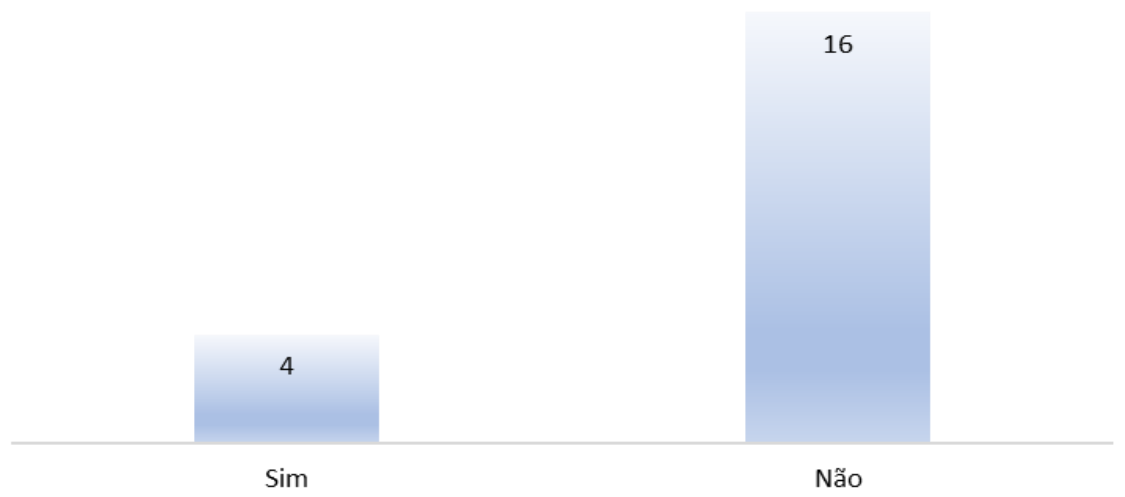

Fonte: Autores (2021).

Essas gestantes informaram que as orientações foram passadas pela enfermeira da unidade básica de saúde, o que é muito aprazível. Pois, dentre a equipe de saúde, o enfermeiro ganha destaque nessa assistência às gestantes, uma vez que a enfermagem se caracteriza em cuidar do ser humano na sua totalidade, seja orientando, supervisionando e/ou auxiliando no que for cabível (Almeida \& Souza, 2016).

As gestantes que não foram esclarecidas sobre a DHEG durante o pré-natal, apontaram para a necessidade de que 
essas informações sejam propagadas, a fim de que haja um cuidado maior quanto a essa patologia. Vemos isso apresentado nas falas a seguir:
G1 - "Sim, pois deixará a gestante ciente e ela poderá se prevenir"
G16 - "Com certeza para ficar ciente sobre os cuidados"
G17 - "Sim, pois pode evitar os riscos"
G20 - "Sim, pois a gestante deve ter todos os cuidados do começo da gestação até o final"

Com isso, vê-se a inevitabilidade em que haja a transmissão de conhecimentos por parte dos profissionais de saúde que atuam na Atenção Básica, para as gestantes que iniciam o pré-natal na unidade, pois, é a partir das informações e orientações oriundas da educação em saúde, que a população exerce mudanças de hábitos e comportamentos, o que resulta em melhor qualidade de vida (Oliveira et al., 2014).

Normalmente, nas consultas de pré-natal, apenas é informado para a gestante sobre as condições da gestação, se está tudo bem ou se é necessário que sejam encaminhadas para o alto risco, sem receberem nenhuma orientação mais precisa. Porém, além de apresentar um papel fundamental na prevenção e detecção precoce de patologias materno-fetais, o pré-natal propicia um momento oportuno para o desenvolvimento da educação em saúde para as gestantes, pois, além de ser um meio de preparação física e psicológica, é também de intenso aprendizado.

Essa educação em saúde proporciona uma interação entre os profissionais e as gestantes, promovendo uma articulação de saberes técnicos e populares, de recursos institucionais e comunitários, com a finalidade de viabilizar a promoção em saúde e facilitar a compreensão do processo gestacional (Correio et al., 2015).

\section{Conclusão}

Por meio do desenvolvimento deste estudo foi possível compreender os conhecimentos das gestantes sobre a DHEG, onde mostrou-se resultados superficiais frente às noções básicas desta patologia, como: definição, sinais e sintomas e complicações para a mãe e para o bebê, elucidando de tal forma, um conhecimento insuficiente, o que é de extrema relevância, visto que o conhecimento auxilia na desconstrução de conceitos errôneos, consequentemente, na prevenção da doença e promoção da saúde.

Observou-se que essas gestantes estão desprovidas de informações essenciais para esse período, sendo necessária uma atuação mais efetiva dos profissionais de saúde da atenção básica, no que diz respeito à promoção de saúde, enfocando possíveis complicações que podem surgir no período gravídico, pois o pouco conhecimento que essas gestantes possuem, são insuficientes para prevenir os agravos na saúde causados pela DHEG. Com isso, nota-se que a Atenção Básica tem um papel fundamental na construção de conhecimentos e promoção da saúde das gestantes, sendo necessário, portanto, que haja uma melhora na assistência, ou seja, um pré-natal de qualidade.

Assim, diante dos resultados apresentados, onde percebe-se limitações frente aos conhecimentos das gestantes pela temática, sugere-se um estudos futuros mais amplo que possam representar um montante maior de gestantes do município, e até mesmo do Estado, bem como, uma maior participação e envolvimento dos profissionais das unidades básicas de saúde com as gestantes, além de elaboração de estratégias e atividades, fortalecendo assim as ações de educação em saúde para as gestantes.

\section{Referências}

Abrahão, A. C. M., Santos, R. C. M., Viana, S. R. G., Viana, S. M. \& Costa, C. S. C. (2020). Atuação do enfermeiro a pacientes portadores de síndrome hipertensiva específica da gestação. Rev. Cient. Esc. Estadual Saúde Pública Goiás “Candido Santiago”, 6(1), 51-63.

Almeida, G. B. S. \& Souza, M. C. M. (2016). O conhecimento da gestante sobre a hipertensão na gravidez. Revista de APS - Atenção Primária à Saúde, 19(3), 396-402. 
Antônio, E. D. A. P., Pereira, T. V. \& Galdino, C. V. (2019). O conhecimento das gestantes sobre síndrome hipertensiva específica da gravidez (SHEG). Saber Digital, 12(1), 1-3.

Antunes, M. B., Dermitto, M. O., Gravena, A. A. F., Padovani, C. \& Pelloso, S. M. (2017). Síndrome hipertensiva e resultados perinatais em gestação de alto risco. Rev Min Enferm, 21, 1-6.

Aragão, J. (2011). Introdução aos estudos quantitativos utilizados em pesquisas científicas. Práxis, 3(6), 60-62.

ARAÚJO, I. F. M., Santos, P. A., Santos, P. A. \& Franklin, T. A. (2017). Síndromes hipertensivas e fatores de risco associados à gestação. Revista de enfermagem UFPE online, 11(10), 4254-4262.

BERHE, A. K., Ilesanmi, A. O., Aimakhu, C. O. \& Bezabih, A. M. (2020). Awareness of pregnancy induced hypertension among pregnant women in Tigray Regional State, Ethiopia. Pan African Medical Journal, 35(71). doi:10.11604/pamj.2020.35.71.19351.

BRASIL. (2016). Importância do pré-natal. Biblioteca Virtual em Saúde. Ministério da Saúde.

BRASIL. (2011). Política nacional de atenção integral à saúde da mulher: princípios e diretrizes. Ministério da Saúde. http://bvsms.saude.gov.br/bvs/publicacoes/politica_nacional_mulher_principios_diretrizes.pdf.

Cassiano, A. N. C., Vitorino, A. B. F., Oliveira, S. I. M., Silva, M. L. C., Sousa, N. M. L. \& Souza, N. L. (2020). Desfechos perinatais em gestantes com síndromes hipertensivas: revisão integrativa. Revista de Enfermagem UFSM, 10(23), 1-20.

Correio, K. D. L., Ramos, A. I. G., Santos, R. L. G., Bushatsky, M. \& Correio, M. B. S. C. B. (2015). Control of cervical cancer: actions taken by nurses based on collective subject discourse. Cuidado é Fundamental, 7(2), 2425-2439.

Gil, A. C. (2017). COMO ELABORAR PROJETOS DE PESQUISA. Atlas, 6. https://docero.com.br/doc/nc0cesv.

Guimarães, J. P., Medeiros, L. G. S., Oliveira, F. C. S. \& Oliveira, S. F. (2014). A prevalência de gestantes portadoras de SHEG que evoluíram para síndrome HELLP em uma maternidade pública. REBES, 4(1), 1-17.

Jacob, L. M. S., Alverne, D. G. B. M., Figueira, M. C. S., Mafetoni, R. R., Pimenta, C. J. L. \& Shimo, A. K. K. (2018). Conhecimentos de Gestantes sobre a Síndrome Hipertensiva Gestacional. Revista Enfermagem Atual, 86(24),

Jacob, L. M. S., Lopes, M. H. B. M. \& Shimo, A. K. K. (2021). Instrumento sobre conhecimento, atitude e prática de gestantes acerca da síndrome hipertensiva gestacional. Rev Rene, 22(60040). https://doi.org/10.15253/2175-6783.20212260040.

Leal, M. C., Szwarcwald, C. L., Almeida, P. V. B., Aquino, E. M. L., Barreto, M. L., Barros, F. \& Victora, C. (2018). Saúde reprodutiva, materna, neonatal e infantil nos 30 anos do Sistema Único de Saúde (SUS). Ciência \& Saúde Coletiva, 23(6), 1915-1928.

Mota, J. F., Almeida, M. S., Magalhães, G. C., Souza, V. C., Silva, J. M. Q. \& Anjos, K. F. (2021). Saberes e experiências de gestantes sobre o autocuidado puerperal e cuidado do/a recém-nascido/a mediante práticas educativas. Rev baiana enferm, 35 .

Oliveira, A. C., Pessoa, R. S., Carvalho, A. M. C. \& Magalhães, R. L. B. (2014) Risk and protection factors for women's health in the prevention of cervical cancer. Rev Rene, 15(2), 240-248.

Oliveira, A. C. M., Santos, A. A., Bezerra, A. R., Barros, A. M. R. \& Tavares, M. C. M. (2016). Fatores maternos e resultados perinatais adversos em portadores de pré-eclâmpsia em Maceió, Alagoas. Arq Bras Cardiol, 106(2), 113-120.

Santana, R. S., Fontes, F. L. L., Carvalho, F. R., Moura, F. F., Duarte, J. M., Cruz, J. F., Gaia, J. O., Silva, T. D., Santos, J. S., Alencar, A. B. B., Sousa, A. V. L., Lima, A. O., Veloso, M. R. B. \& Silva, A. S. (2019). Importância do conhecimento sobre sinais e sintomas da pré-eclâmpsia para implementação dos cuidados de Enfermagem. Revista Eletrônica Acervo saúde, 11(15).

Silva, E. C., Silva, N. C. D. L., Silva, A. E. G., Campos, R. L. O., Santana, M. R., Café, L. A., Almeida, P. M. O., Oliveira, S. M., Gomes, A. S. \& Silva, A. T. C. S. G. (2021). Atuação do enfermeiro na prevenção das síndromes hipertensivas na gestação no âmbito da atenção básica. Revista Eletrônica de Saúde, 13(12).

Silva, E. F., Cordova, F. P., Chachamovich, J. L. R. \& Záchia, S. A. (2011). Percepções de um grupo de mulheres sobre a doença hipertensiva específica da gestação. Rev Gaúcha Enferm., 2(32), 316-322..

Silva, M. L. \& Emmendoerfer, M. L. (2014). Resenha Bibliográfica - Pesquisa Qualitativa em Administração: Fundamentos, Métodos e usos no Brasil. Organizações em contexto, 10(20), 453-458.

Sociedade brasileira de cardiologia. (2016). $7^{\circ}$ Diretriz Brasileira de Hipertensão Arterial. Portal Cardiol. http://publicacoes.cardiol.br/2014/diretrizes/2016/05_HIPERTENSAO_ARTERIAL.pdf.

Sociedade brasileira de cardiologia. (2021) Diretrizes Brasileiras de Hipertensão - 2020. Arq. Bras. Cardiol., 116(3), 516-658. http://departamentos.cardiol.br/sbc-dha/profissional/pdf/Diretriz-HAS-2020.pdf.

Soeiro, R. E., Bedrikow, R., Ramalho, B. D. S., Niederauer, A. J. S., Souza, C. V., Previato, C. S., Martins, D. B., Dias, T. M., Freitas, A. R. R. \& Dimarzio, G. (2020). Atenção Primária à Saúde e a pandemia de COVID-19: reflexão para a prática. Interamerican journal of medicina and health, (3).

Teixeira, G. A., Carvalho, J. B. L., Sena, A. V., Morais, P. C. \& Alves, T. R. M. (2017). Características maternas de partos prematuros. Revista Enfermagem Atual, 81(19). https://doi.org/10.31011/reaid-2017-v.81-n.19-art.317.

Thuler, A. C. M. C., Wall, M. L., Benedet, D. C. F., Souza, S. R. R. K. \& Souza, M. A. R. (2018). Medidas preventivas das síndromes hipertensivas da gravidez na atenção primária. Revista de enfermagem UFPE online, 12(4), 1060-1071. 